\title{
Spatially resolved stellar populations with SAMI
}

\author{
Nicholas Scott ${ }^{1,2}$ and the SAMI team \\ ${ }^{1}$ Sydney Institute for Astronomy, The University of Sydney, 44 Rosehill Street, Redfern, NSW, \\ 2016 Australia \\ ${ }^{2}$ ARC Centre of Excellent for All-Sky Astronomy (CAASTRO) \\ email: nscott@physics.usyd.edu.au
}

\begin{abstract}
Using data from the SAMI Galaxy Survey we measure azimuthally averaged stellar age and metallicity profiles for $\sim 500$ galaxies, using both luminosity-weighted Lick indices and mass-weighted full spectral fitting. We find a weak trend for steeper (i.e. more negative) metallicity gradients in more massive galaxies, however, below stellar masses $\sim 10^{10.5} \mathrm{M}_{\odot}$, the scatter in metallicity gradient increases dramatically.
\end{abstract}

Keywords. galaxies: abundances - galaxies: evolution - galaxies: formation

\section{Introduction}

The spatial distribution of the stellar population of a galaxy holds important clues as to its formation and assembly. Age gradients can reveal when and where new gas is accreting onto a galaxy. Metallicity gradients can be used to distinguish between stars that formed in-situ and stars that have been accreted onto a galaxy. In particular, a change in metallicity gradient, from relatively steep to shallow or flat is a strong indicator of a change from predominantly in-situ (or monolithic collapse-like) to accreted stellar populations (e.g. Font et al. 2011).

\section{Sample and Methodologies}

The Sydney-AAO Multi-object Integral-field spectrograph (SAMI) is an innovative new instrument on the $4 \mathrm{~m}$ Anglo-Australian Telescope. It uses a fused-fibre "hexabundle" design to take simultaneous integral-field spectroscopy of up to 13 targets. SAMI provides simultaneous blue wavelength coverage from 3800 to $5700 \AA$ at $\mathrm{R} \sim 1700$ and red wavelength coverage from 6300 to $7500 \AA$ at $\mathrm{R} \sim 4500$. The SAMI Galaxy Survey (SGS) is a large program with SAMI targeting $\sim 3400$ galaxies in the redshift range $0.01<z<0.1$, spanning a broad range of stellar mass and local environmental density. This study uses the first $\sim 20$ per cent of observations from the SGS.

We extract stellar population information by radially binning our spatially resolved spectroscopy. For each radial bin we measure stellar ages, t, and metallicities, $[\mathrm{Z} / \mathrm{H}]$, through two complementary techniques:

(a) Luminosity-weighted age and metallicity. We measure 5 Lick (Worthey et al. 1994) spectral indices, $\mathrm{H} \beta, \mathrm{Mgb}, \mathrm{Fe} 5015, \mathrm{Fe} 5270$ and Fe5335. Following Kuntschner et al. (2010) we use the stellar population models of Schiavon (2007) to derive $[\mathrm{Z} / \mathrm{H}]$ and t.

(b) Mass-weighted age and metallicity. Following McDermid et al. (2014), we fit weighted combinations of Single Stellar Population (SSP) model template spectra from Vazdekis et al. (2010) to our observations. From the relative weights assigned to each template we then determine the mean mass-weighted $[\mathrm{Z} / \mathrm{H}]$ and $\mathrm{t}$. 


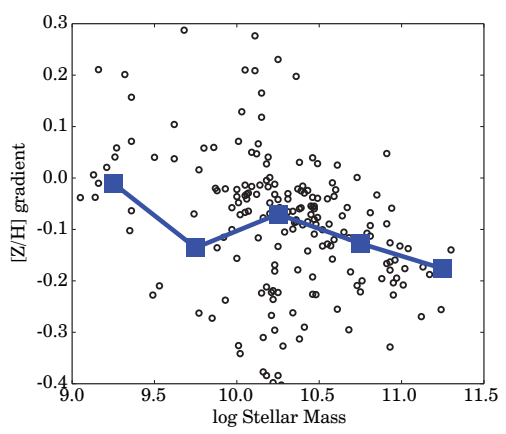

Figure 1. Mass-weighted $[\mathrm{Z} / \mathrm{H}]$ gradient vs. galaxy stellar mass. The large points and solid line indicate the mean values within 5 narrow bins of stellar mass. Below $M_{\star} \simeq 10^{10.5} M_{\odot}$ the range of gradients increases dramatically. Above this mass we find a weak trend of increasing steep (i.e. more negative) gradients with increasing stellar mass.

\section{Results}

We are able to extract age and metallicity gradients for $\gtrsim 75$ per cent of galaxies in our partial SGS sample. The success rate for the luminosity-weighted and mass-weighted techniques is comparable. For a subset of $\sim 200$ of our galaxies, for which we measure $[\mathrm{Z} / \mathrm{H}]$ in multiple radial bins with small uncertainties, and for which we have obtained reliable effective radii, $\mathrm{R}_{e}$, and stellar masses, $\mathrm{M}_{\star}$, we derive metallicitiy gradients, $\Delta$ $[\mathrm{Z} / \mathrm{H}]=\Delta[\mathrm{Z} / \mathrm{H}] / \Delta \mathrm{R}_{e}$ (Figure 1 ). We find that $\Delta[\mathrm{Z} / \mathrm{H}]$ is typically negative, i.e. metallicity decreases with galactic radius. We find a weak trend of increasingly steep (i.e. more negative $\Delta[\mathrm{Z} / \mathrm{H}]$ ) in more massive galaxies. Below stellar masses $\sim 10^{10.5}$ the scatter in this relation increases dramatically.

\section{Outlook}

With the full SGS sample of $\sim 3400$ galaxies we will be able to derive age and metallicity profiles for thousands of galaxies out to $1-2 \mathrm{R}_{e}$, an order of magnitude more than any previous study. For the first time, we will be able to explore the dependence of stellar population gradients on stellar mass and environmental density in a large sample of galaxies, which will provide important constraints for the upcoming generation of cosmological galaxy formation models.

\section{Acknowledgements}

This research was conducted by the Australian Research Council Centre of Excellence for All-sky Astrophysics (CAASTRO), through project number CE110001020.

\section{References}

Font, A. S., McCarty, I. G., Crain, R. A., Theuns, T., Schaye, J., Wiersma, R. P. C., \& Dalla Vecchia, C., , 2010, MNRAS, 416, 2802

Kuntschner, H., et al., 2010, MNRAS, 408, 97

McDermid, R., et al., 2014, MNRAS, submitted

Schiavon, R. P., 2007, ApJS, 171, 146

Vazdekis, A., et al., 2010, MNRAS, 404, 1639

Worthey, G., Faber, S. M., Gozalez, J. J., \& Burstein, D., 1994, ApJS, 94, 687 